\section{Original Article}

Check for updates

\section{OPEN ACCESS}

Received: Oct 29, 2018

Accepted: Feb 20, 2019

Correspondence to

Miaomiao Sheng

Laboratory of Molecular Genetics of Aging \&

Tumor, Medical School, Kunming University of

Science and Technology, Chenggong Campus,

727 South Jingming Road, Kunming,

Yunnan 650500, China.

E-mail: shengmm@aliyun.com

*These authors contributed equally to this work.

C 2019 Korean Breast Cancer Society This is an Open Access article distributed under the terms of the Creative Commons Attribution Non-Commercial License (https:// creativecommons.org/licenses/by-nc/4.0/) which permits unrestricted non-commercial use, distribution, and reproduction in any medium, provided the original work is properly cited.

ORCID iDs

Wenzhu Dai (ID

https://orcid.org/0000-0001-7953-4969 Jixiang $\mathrm{He}$ (D)

https://orcid.org/0000-0002-6747-2665 Ling Zheng (1D)

https://orcid.org/0000-0002-5432-1569 Mingyu Bi (D)

https://orcid.org/0000-0002-4231-2091

Fei Hu (D)

https://orcid.org/0000-0002-5761-8691 Minju Chen (D)

https://orcid.org/0000-0002-8835-1977

Heng Niu (iD)

https://orcid.org/0000-0002-7071-6763

\title{
miR-148b-3p, miR-190b, and miR-429 Regulate Cell Progression and Act as Potential Biomarkers for Breast Cancer
}

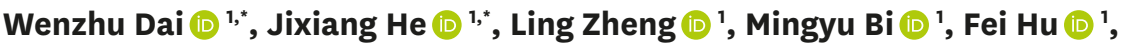

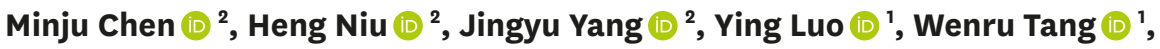 \\ Miaomiao Sheng (1) ${ }^{1}$
}

'Laboratory of Molecular Genetics of Aging \& Tumor, Medical School, Kunming University of Science and Technology, Yunnan, China

${ }^{2}$ Department of Mammary Gland and Thyroid Disease, First People's Hospital of Yunnan Province, Yunnan, China

\section{ABSTRACT}

Purpose: Breast cancer is the most frequently diagnosed malignancy in women worldwide. MicroRNAs (miRNAs) are thought to serve as potential biomarkers in various cancers, including breast cancer.

Methods: We evaluated the miRNA expression profiles in 1,083 breast cancer samples and 104 normal breast tissues from The Cancer Genome Atlas database. We used the edgeR package of $\mathrm{R}$ software to analyze the differentially expressed miRNAs in normal and cancer tissues, and screened survival-related miRNAs by Kaplan-Meier analysis. A receiver operating characteristic curve was generated to evaluate the accuracy of these miRNAs as molecular markers for breast cancer diagnosis. Furthermore, the functional role of these miRNAs was verified using cell experiments. Targets of candidate miRNAs were predicted using 9 online databases, and Gene Ontology (GO) functional annotation and pathway analyses were conducted using Database for Annotation, Visualization and Integrated Discovery online tool. Results: A total of 68 miRNAs showed significantly different expression patterns between the groups $(p<0.001)$, and 13 of these miRNAs were significantly associated with poor survival $(p<0.05)$. Three miRNAs with high specificity and sensitivity, namely, miR-148b-3p, miR$190 \mathrm{~b}$, and miR-429, were selected. In vitro experiments showed that the overexpression of these 3 miRNAs significantly promoted the proliferation and migration of MDA-MB-468 and T47D cells and reduced the apoptosis of T47D cells. GO and pathway enrichment analyses revealed that the targets of these dysregulated miRNAs were involved in many critical cancerrelated biological processes and pathways.

Conclusion: The miR-148b-3p, miR-190b, and miR-429 may serve as potential diagnostic and prognostic markers for breast cancer. This study demonstrated the roles of these 3 miRNAs in the initiation and progression of breast cancer.

Keywords: Biological marker; Biological phenomenon; Breast neoplasm; MicroRNA

\section{INTRODUCTION}

Breast cancer, the most common malignant disease in women, is the second leading cause of cancer-related death $[1,2]$. According to the latest statistical report in China, the number 
Jingyu Yang (iD

https://orcid.org/0000-0002-6376-3983 Ying Luo iD

https://orcid.org/0000-0002-5366-8704

Wenru Tang (iD)

https://orcid.org/0000-0002-7231-037X

Miaomiao Sheng (iD

https://orcid.org/0000-0001-6341-8939

Funding

This work was supported by the National Natural Science Foundation of China (grant number 81560451).

\section{Conflict of Interest}

The authors declare that they have no competing interests.

\section{Author Contributions}

Conceptualization: Tang W, Sheng M; Data curation: Dai W, He J, Tang W; Formal analysis: Dai W, He J, Tang W; Funding acquisition: Dai W; Investigation: Zheng L, Bi M, Hu F; Resources: Chen M, Niu H, Yang J; Validation: Sheng M; Visualization: Dai W, He J; Writing - original draft: Dai W, He J, Sheng M; Writing review \& editing: Luo $Y$, Tang $W$. of new breast cancer incidences and related deaths in China each year accounts for $12.2 \%$ and $9.6 \%$ cases in the world, respectively [2]. About 210,000 new breast cancer cases are reported in China every year, and the rate is increasing every year by $2 \%$. An estimated 2.5 million people will be suffering from breast cancer in China by 2021 [2]. Although the improvement in the early examination and diagnosis techniques and advances in surgery and comprehensive adjuvant therapy have significantly improved the prognosis of patients, the overall survival (OS) rate is not ideal. Accumulating evidences indicate that the early diagnosis and treatment result in favorable prognoses [3]. Therefore, it is crucial to identify novel diagnostic biomarkers and prognostic factors with high sensitivity and specificity to meet the clinical requirements for breast cancer.

MicroRNAs (miRNAs) are endogenous small-noncoding RNAs of 18-23 nucleotides that play a significant role in the regulation of gene expression [4]. miRNAs bind to the $3^{\prime}$-untranslated region of the target messenger RNAs (mRNAs) and degrade or repress their translation. Numerous studies have revealed that miRNAs perform pivotal functions in diverse biological processes such as cell growth, differentiation, metabolism, and apoptosis [5]. Moreover, miRNAs are involved in multiple cancer-related cell signaling pathways, including those associated with breast cancer [6]. The aberrant expression of miRNAs in breast cancer has been previously reported and these may act as either tumor suppressors or oncogenes [7], suggestive of their potential role in the diagnosis and prognosis of breast cancer [8]. However, most studies have used microarrays or real-time quantitative polymerase chain reaction (RT-qPCR), which are limited in terms of range of detection. Furthermore, the small sample size and lack of sensitivity and specificity of candidate miRNAs have limited the clinical application of these biomarkers [9]. Thus, it is essential to identify new miRNAs and understand their mechanism of action in the control of various steps involved in tumor malignancy and molecular pathways involved in breast cancer progression.

The Cancer Genome Atlas (TCGA) is a large database of cancer and tumor genetic mapping programs sponsored by the U.S. government and includes gene expression, epigenome, non-coding RNA expression, and clinical data. In the present study, we first identified the differentially expressed miRNAs between breast cancer and normal tissues through the analysis of TCGA database. Thirteen miRNAs that were significantly associated with patient survival were obtained and of these, 3 miRNAs (miR-148b-3p, miR-190b, and miR-429) were eventually used as a signature for the diagnosis of breast cancer. The effects of these 3 miRNAs on biological behaviors of breast cancer cells were also analyzed.

\section{METHODS}

\section{Data collection}

The miRNA-Seq data sets of 1,083 breast cancer and 104 normal samples were collected from TCGA (http://cancergenome.nih.gov/). Clinical information of the samples in TCGA was matched with the data of miRNAs. There were no concerns of ethical issues, as the data were retrieved from TCGA. We extracted the expression matrix of miRNAs using Perl Script, and the edgeR package of R software (R Foundation, Vienna, Austria) was used to detect the differentially expressed miRNAs and their relative fold change. Survival-related miRNAs were screened by Kaplan-Meier analysis and a receiver operating characteristic (ROC) curve was generated to calculate the area under the curve (AUC) at 95\% confidence interval $(95 \% \mathrm{CI})$. 


\section{Bioinformatic prediction of miRNA target genes and enrichment analysis}

The potential targets of candidate miRNAs were predicted using 9 online databases, including miRDB (http://mirdb.org/), miRTarBase (http://mirtarbase.mbc.nctu.edu.tw/), TargetScan (http://www.targetscat.org/vert_72/), RNA22 (https://cm.jefferson.edu/rna22/Precomputed/ OptionController?species=HomoSapiens\&type=mRNA\&version=MB21E78v2), PicTar (https:// pictar.mdcberlin.de/), Tarbase (http://carolina.imis.athenainnovation.gr/diana_tools/web/ index.php?r=tarbasev8\%2Findex), PolymiRTSe (http://compbio.uthsc.edu/miRSNP/search. php), miRBase (http://www.mirbase.org/), and MiRNA (http://starbase.sysu.edu.cn/targetSite. php). Only the target genes predicted by at least 4 databases were chosen. Gene Ontology (GO) functional annotation and pathway analyses were conducted with Database for Annotation, Visualization and Integrated Discovery online tool (https://david.ncifcrf.gov) and visualized by Cytoscape 3.4.0 software (Institute for Systems Biology, Seattle, USA).

\section{Clinical specimens and cell lines}

The study included 75 patients with breast cancer that underwent surgery at the First People's Hospital of Yunnan Province from 2015 to 2017. The breast cancer tissues and adjacent normal tissues were confirmed by a pathologist. Tissue samples were immediately stored in liquid nitrogen after the surgery. The protocol was approved by the Ethical Committee of First People's Hospital of Yunnan Province (No. 2015JC006). This work was carried out in accordance with The Code of Ethics of the World Medical Association (Declaration of Helsinki) and informed consent was obtained from all human subjects. Six human breast cancer cell lines (MDA-MB-468, MDA-MB-231, MCF-7, T47D, SK-BR-3, and HCC-1806) and 1 normal mammary epithelial cell line (MCF-10A) were purchased from the Peking Union Medical College Cell Culture Center (Beijing, China) and cultured at $37^{\circ} \mathrm{C}$ in a humidified incubator with 5\% $\mathrm{CO}_{2}$. MDA-MB-468, MDA-MB-231, and MCF-7 cells were cultured in Dulbecco's modified Eagle's medium (DMEM; C11995500BT, Thermo Fisher Scientific, Waltham, USA) supplemented with 10\% fetal bovine serum (FBS, 10099141, Thermo Fisher Scientific), while T47D, SK-BR-3, and HCC-1806 cells were maintained in Roswell Park Memorial Institute-1640 medium (C11875500BT, Thermo Fisher Scientific) supplemented with 10\% FBS. MCF-10A cells were cultured in DMEM/F12 (12500-062, Thermo Fisher Scientific) medium supplemented with 10\% horse serum (HyClone, Utah, USA), $1 \mathrm{mg} / \mathrm{mL}$ epidermal growth factor (Merck KGaA, Darmstadt, Germany), $1 \mathrm{mg} / \mathrm{mL}$ cholera toxin (Merck $\mathrm{KGaA}$ ), $20 \mathrm{mg} / \mathrm{mL}$ insulin (Merck KGaA), and $1 \mathrm{mg} / \mathrm{mL}$ hydrocortisone (Merck KGaA).

\section{RNA extraction and stem-loop RT-qPCR for the detection of miRNAs}

Total RNA from tissues and cell lines was extracted using TRIzol Reagent (T9424, Merck $\mathrm{KGaA}$ ) according to the manufacturer's instructions. Single-stranded complementary DNA was synthesized from RNA using GoScript ${ }^{\mathrm{TM}}$ Reverse Transcription System (Promega, Wisconsin, USA). For qPCR, a specific forward primer was designed for each miRNA and reverse primers were targeted to the stem-loop sequence. Expression levels of miRNAs were analyzed by qPCR using SYBR Green Master Mix (Roche, Basel, Switzerland), and U6 was used as an internal control. The expression level of each gene was calculated and normalized using the $2^{-\Delta \Delta c t}$ method relative to U6 expression. The primer sequences used were as follows: hsa-U6 forward, 5'-CTCGCTTCGGCAGCACA-3' and reverse, 5'-AACGCTTCACGAATTTGCGT-3'; hsa-miR-148b-3p forward, 5'-TCTCTCCAGTCTACTCAGTGCATC-3' and reverse, 5'-TATGGTTTTGACGACTGTGTGAT-3'; hsa-miR-190b forward, 5'-GGCGACGAGTGATATGTTTGATAT-3' and reverse 5'-TATGGTTGTTCACGACTCCTTCAC-3'; and hsa-miR-429 forward, 5'-CCGCGCTAATACTGTCTGG-3' and reverse 5'-GTGCACGCTCCGAGGT-3'. 


\section{Transfection}

Cells were transfected with $50 \mathrm{nM}$ of miRNA mimic or $100 \mathrm{nM}$ miRNA inhibitor and their corresponding negative controls (RiboBio, Guangzhou, China) using TransIntro EL transfection reagent (TransGen Biotech, Beijing, China), as per the procedures described in the manufacturer's instructions.

\section{Cell proliferation assay}

Cells were seeded in 24-well plates and after 48 hours of transfection, 5-ethynyl-2'deoxyuridine (EdU) was used to measure the proliferation of breast cancer cells. Cells were exposed to $25 \mathrm{mM}$ of EdU (RiboBio) for 2 hours at $37^{\circ} \mathrm{C}$ and fixed with $4 \%$ paraformaldehyde in phosphate-buffered saline (PBS). After permeabilization with $0.5 \%$ Triton-X, the cells were reacted with Apollo reaction cocktail (RiboBio), for 30 minutes, followed by staining with Hoechst 33342 for 30 minutes and visualization with inverted microscopy (TS100, Nikon, Tokyo, Japan). Image Pro Plus 6.0 software (Media Cybernetics, Washington, D.C., USA) was used to count the number of cells and to evaluate cell proliferation. Percentage of EdUpositive cells was calculated as (EdU-positive cells/Hoechst-stained cells) $\times 100 \%$.

\section{Apoptosis analysis}

The apoptotic rate of cells was measured using Annexin V-fluorescein isothiocyanate Apoptosis Detection Kit (11988549001, Roche) according to the manufacturer's instructions. Cells were seeded in 12-well plates and transfected with miRNA mimics or inhibitor. After 48 hours, the harvested cells were incubated in a buffer supplemented with $1 \mu \mathrm{L}$ Annexin V and $2 \mu \mathrm{L}$ propidium iodide for 30 minutes at $15^{\circ} \mathrm{C}$ and analyzed by flow cytometry (Becton Dickinson, Franklin Lakes, USA).

\section{Western blot analysis}

Protein lysates were separated using sodium dodecyl sulfate polyacrylamide electrophoresis gels and transferred onto polyvinylidene fluoride membranes (Merck KGaA), followed by incubation with primary antibodies, including poly (ADP-ribose) polymerase (PARP; CST, 9542S, 1:1000) and glyceraldehyde-3-phosphate dehydrogenase (ABclonal, AC001, 1:1000) overnight at $4^{\circ} \mathrm{C}$. The membranes were washed thrice with Tris-buffered saline and Tween-20 for 10 minutes and incubated with secondary antibodies for 2 hours at room temperature. The protein bands were examined with Immobilon Western Chemiluminescent reagent (Merck KGaA) and images were captured with a chemiluminescence imaging system (Tanon 5200, Shanghai, China).

\section{Wound healing assay}

Wound healing assay was used to assess the migratory ability of tumor cells. Cells $\left(3 \times 10^{5}\right.$ cells/well) were seeded in a 12-well plate and transfected with miRNA mimics or inhibitor after overnight incubation. After 6 hours, the cell layer was scratched using a pipette tip and washed twice with PBS. These cells were cultured for 24 hours with $1 \%$ low serum medium. Images of cell morphology were captured at initiation time under Olympus microscope (Olympus Corporation, Tokyo, Japan). The migratory ability was quantified and normalized by relative gap distance.

\section{Transwell assays}

After transfection for 6 hours, the cells were seeded in $6.5 \mathrm{~mm}$ transwell chambers with $8 \mu \mathrm{m}$ pores (3422, Corning Incorporated, New York, USA). The upper chambers were filled with $200 \mu \mathrm{L}$ of transfected cells $\left(8 \times 10^{4}\right.$ cells), and the lower chambers were filled with $500 \mu \mathrm{L}$ of 
complete medium. After 24 hours of incubation, the non-migratory cells that remained on the upper surface were removed with a cotton swab. The migrated cells on the lower surface of the membrane insert were fixed with $4 \%$ paraformaldehyde in PBS and stained with $0.1 \%$ crystal violet. The number of cells in the lower chamber was counted with light microscopy.

\section{Statistical analyses}

Data were shown as the mean \pm standard deviation (SD). All statistical analyses were conducted using SPSS version 20 software (IBM Corp., Armonk, USA), GraphPad Prism 7 (GraphPad Software, La Jolla, USA), and R language (version 3.3.1; http://www.r-project.org/; $\mathrm{R}$ Foundation). A value of $p<0.05$ was considered statistically significant. All experiments were performed at least thrice with triplicate samples.

\section{RESULTS}

\section{Selection of candidate miRNAs}

As shown in the flow chart (Figure 1A), 1,083 breast cancer samples and 104 normal control breast tissue samples from TCGA database were analyzed. A total of 68 miRNAs showed significantly different expression patterns between groups (Supplementary Table 1). Of these, 50 miRNAs were downregulated and 18 miRNAs showed upregulated expression in breast cancer specimens. In Kaplan-Meier analysis, 13 miRNAs were significantly associated with poor survival (Figure 1B and Supplementary Figure 1). The ROC curve is a well-recognized statistical method widely used for the identification of disease prediction accuracy. Thirteen miRNAs were subjected to ROC curve analysis, and finally 3 miRNAs with an AUC value higher than 0.8 were selected. These included miR-148b-3p (AUC = 0.852;

A

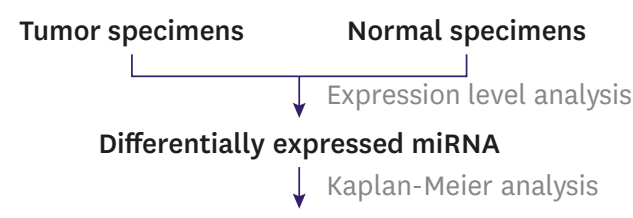

miRNA associated with overall survival

$\downarrow$ ROC analysis

Candidate miRNA
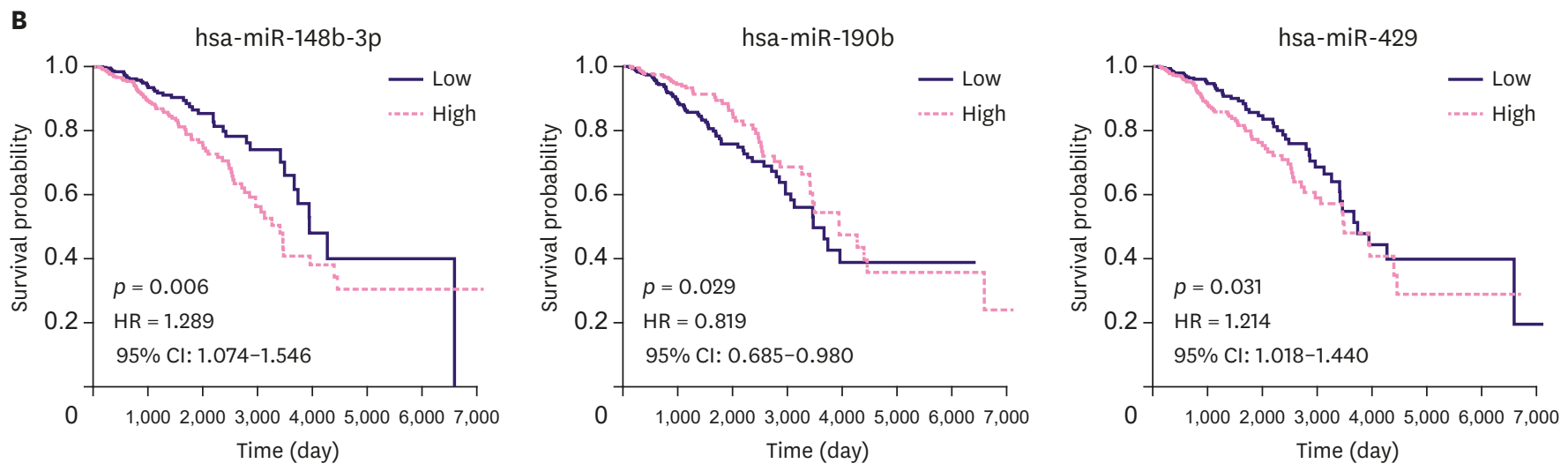

Figure 1. Identification of the 3 miRNAs. (A) Overall workflow of the study. (B) Kaplan-Meier survival curves showing different overall survival in groups of patients with low and high miRNAs expression. (C) ROC curves analysis for miR-148b-3p, miR-190b, and miR-429 differentiating tumor specimens from normal specimens. miRNA = microRNA; $\mathrm{HR}=$ hazard ratio; $\mathrm{Cl}$ = confidence interval; $\mathrm{AUC}=$ area under the curve; $\mathrm{ROC}=$ receiver operating characteristic. (continued to the next page) 

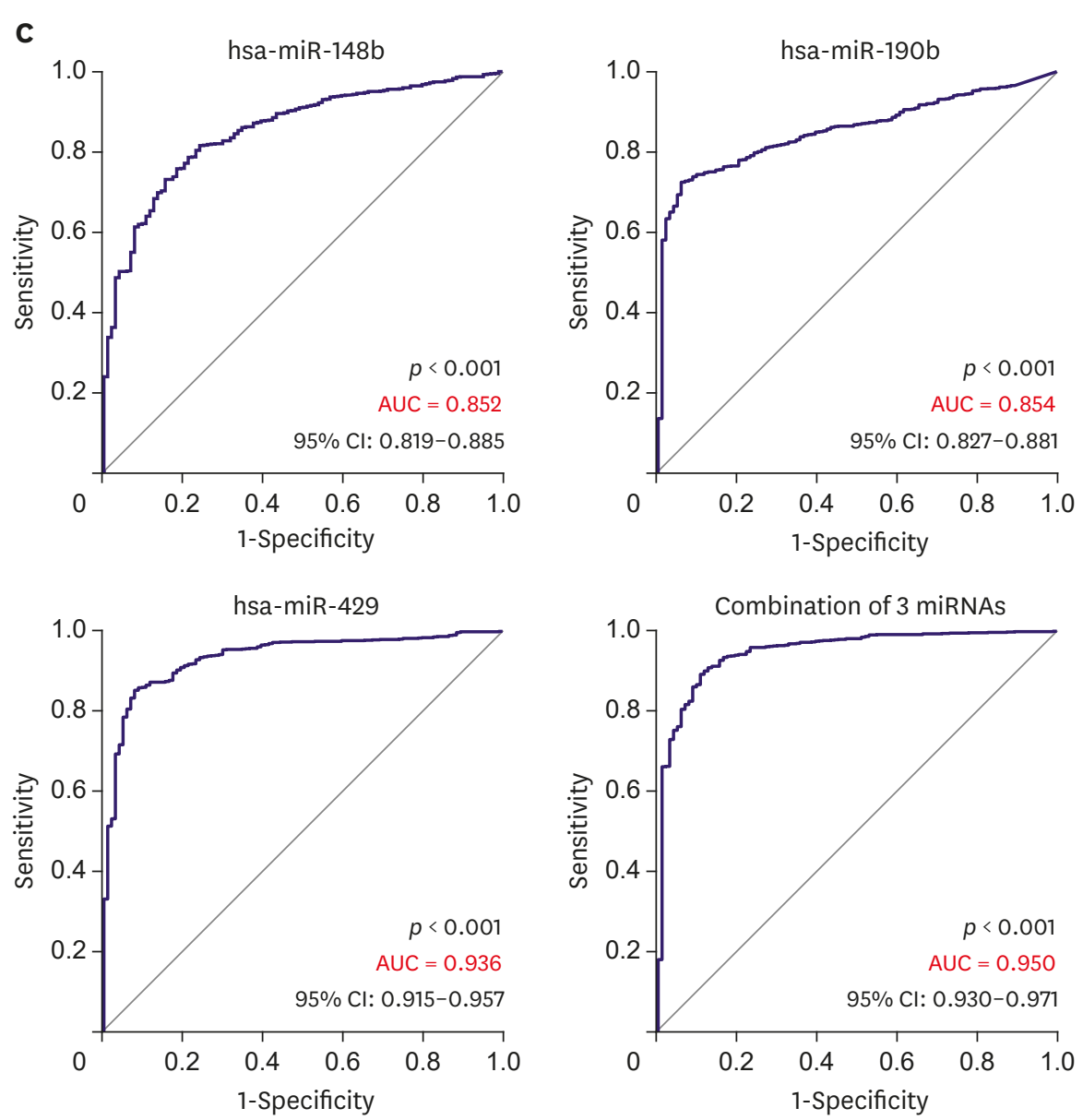

Figure 1. (Continued) Identification of the 3 miRNAs. (A) Overall workflow of the study. (B) Kaplan-Meier survival curves showing different overall survival in groups of patients with low and high miRNAs expression. (C) ROC curves analysis for miR-148b-3p, miR-190b, and miR-429 differentiating tumor specimens from normal specimens.

miRNA = microRNA; $\mathrm{HR}=$ hazard ratio; $\mathrm{Cl}=$ confidence interval; $\mathrm{AUC}=$ area under the curve; $\mathrm{ROC}=$ receiver operating characteristic.

95\% CI, 0.819-0.885; $p<0.001$ ), miR-190b (AUC = 0.854; 95\% CI, 0.827-0.881; $p<0.001$ ), and miR-429 (AUC = 0.936; 95\% CI, 0.915-0.957; $p<0.001$ ) (Figure 1C). To improve the predictive value of miRNAs, we constructed a binary logistic regression model to evaluate the combination of these 3 miRNAs. The miRNA signature showed improved accuracy for the prediction of breast cancer than each miRNA alone with an AUC value of 0.950 (95\% CI, $0.930-0.971, p<0.001$ ) (Figure 1C), while the diagnostic sensitivity and specificity reached $89.4 \%$ and $89.2 \%$, respectively. Taken together, these results indicate that the 3 miRNAs exhibited reliable performance in the diagnosis of breast cancer.

\section{Expression of miR-148b-3p, miR-190b, and miR-429 was enhanced in breast cancer tissues and cell lines}

miR-148b-3p, miR-190b, and miR-429 showed high expression in TCGA database (Figure 2A and Supplementary Figure 2). We examined the expression levels of these 3 miRNAs using RT-qPCR in breast cancer samples. The pathological features of patients are presented in Table 1. The outcome showed that the expression of the 3 miRNAs was higher in breast cancer tissues than in normal controls. Although no significant difference was observed between the groups, the changing trend of the 3 miRNAs was consistent with the observations from TCGA database (Figure 2B). Moreover, miR-148b-3p, miR-190b, and miR-429 expression was 
A
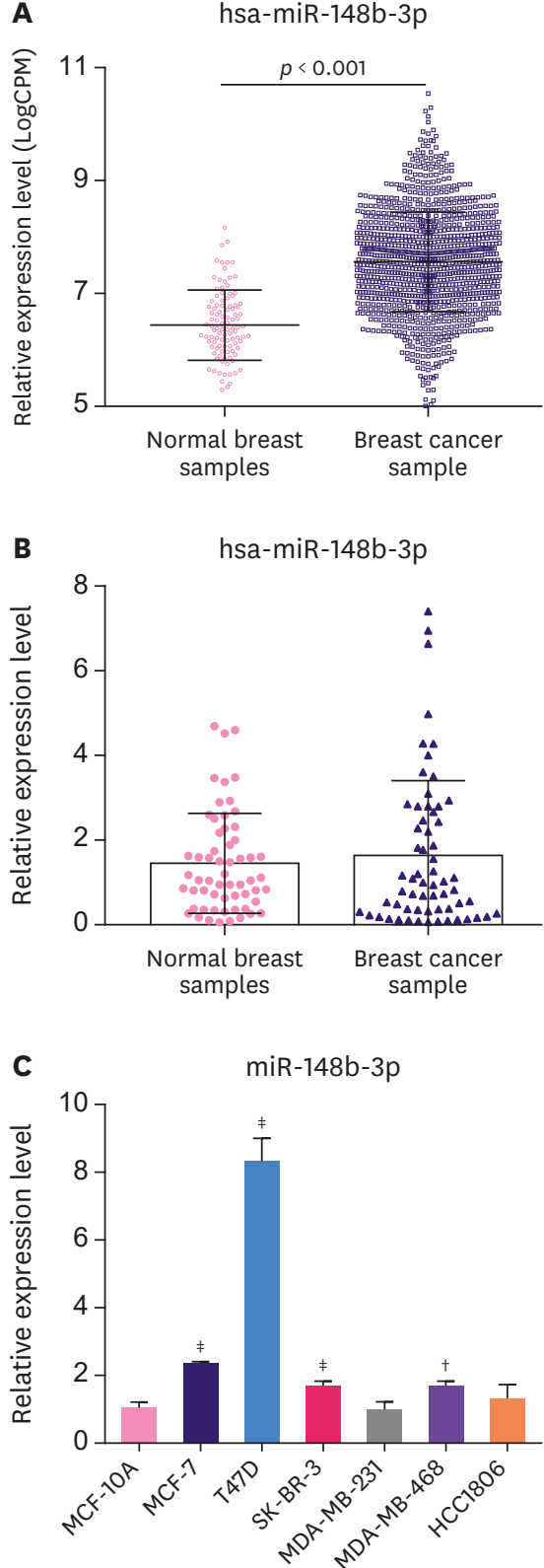

hsa-miR-190b

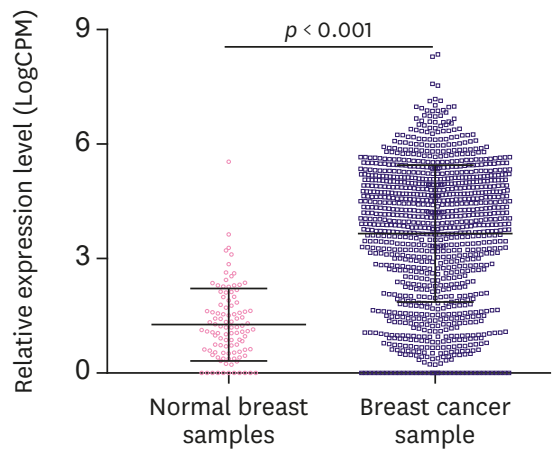

hsa-miR-190b

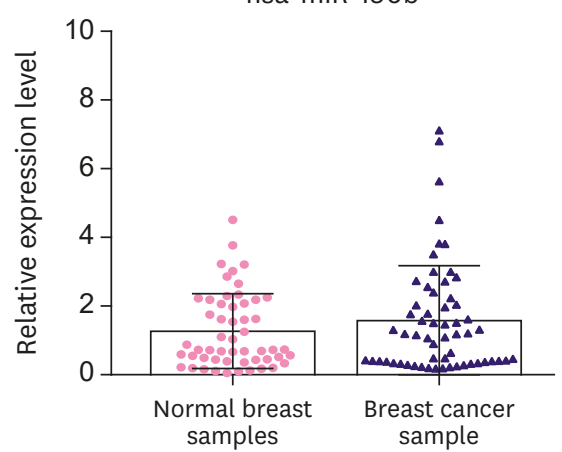

miR-190b

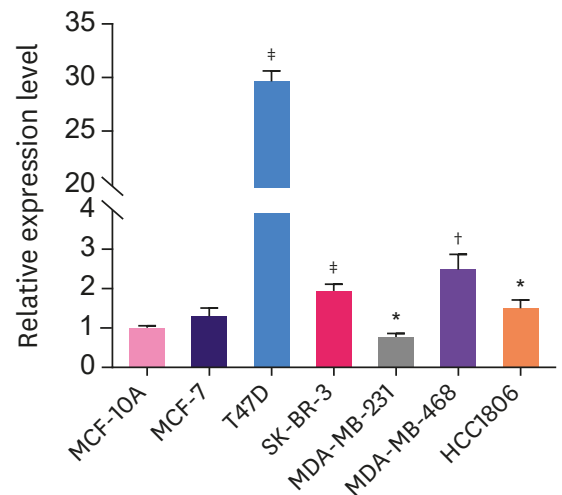

hsa-miR-429

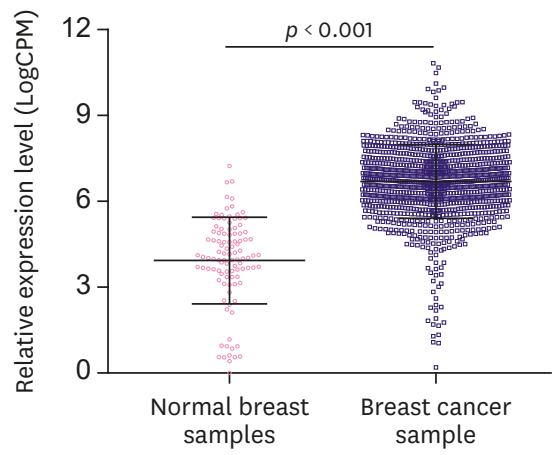

hsa-miR-429

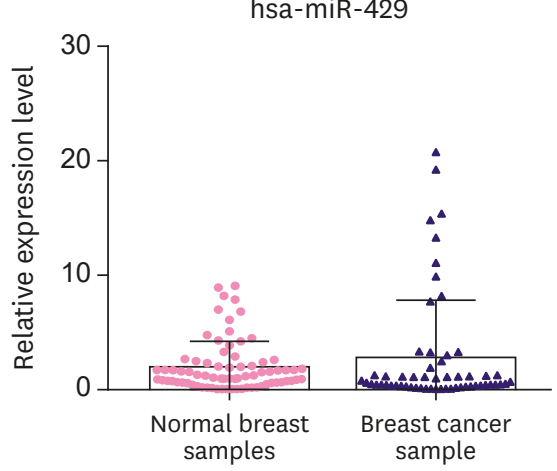

$\operatorname{miR}-429$

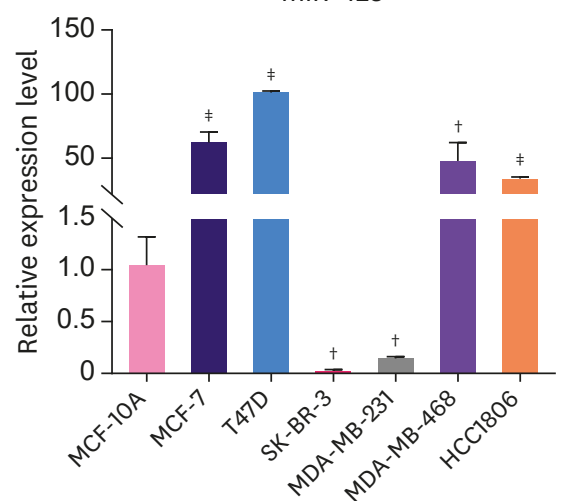

Figure 2. The expression of miR-148b-3p, miR-190b, and miR-429 was enhanced in breast cancer tissues and cell lines. (A) Expression levels of the 3 miRNAs in TCGA. (B) Expression levels of the 3 miRNAs were examined in breast cancer samples. (C) Expression levels of the 3 miRNAs were examined in 7 cell lines. TCGA = The Cancer Genome Atlas; miRNA = microRNA.

${ }^{*} p<0.05,{ }^{\dagger} p<0.01,{ }^{\ddagger} p<0.001$ vs. MCF-10A using 1-way analysis of variance.

further confirmed in breast cancer cell lines. As shown in Figure 2C, miR-148b-3p and miR-190b were overexpressed in the 4 breast cancer cell lines (MCF-7, T47D, SK-BR-3, MDA-MB-468, and HCC-1806) as compared with the normal mammary epithelial cell line (MCF-10A) but were downregulated in MDA-MB-231 cells (Figure 2C). miR-429 expression was significantly upregulated in MCF-7, T47D, MDA-MB-468, and HCC-1806 cells but was downregulated in SK-BR-3 and MDA-MB-231 cells (Figure 2C). These observations suggest that the biological and clinical implication of miRNAs may differ among breast cancer subtypes; hence, we carried out Kaplan-Meier analysis in different breast cancer subtypes based on TCGA database. We found that patients with high miR-148b-3p and miR-429 expression levels had worse 
OS than those with low-level expression in all subtypes (Supplementary Figure 3A and B). However, in luminal A, basal-like, and normal breast-like groups, high expression of miR-190b correlated with better OS. On the other hand, luminal B and human epidermal growth factor receptor 2 (HER2) groups showed high expression of miR-190b that correlated with worse OS (Supplementary Figure 3C).

\section{Effects of miR-148b-3p, miR-190b, and miR-429 on breast cancer cell proliferation}

To investigate the influence of miR-148b-3p, miR-190b, and miR-429 on breast cancer cell proliferation, T47D and MDA-MB-468 cells were treated with these miRNA mimics and inhibitors and the expression of the 3 miRNAs was verified by RT-qPCR (Supplementary Figure 4). Cell proliferation was detected with EdU assay. As shown in Figure 3A, the proliferation ability of T47D cells significantly increased after the overexpression of miR148b-3p, miR-190b, and miR-429, but opposite effects were observed after the silencing of these miRNAs. In MDA-MB-468 cells, these miRNA mimics could significantly promote proliferation and the inhibitors suppressed cell proliferation, although the results observed with miR-148b-3p and miR-429 showed no statistical difference (Figure 3B).

A
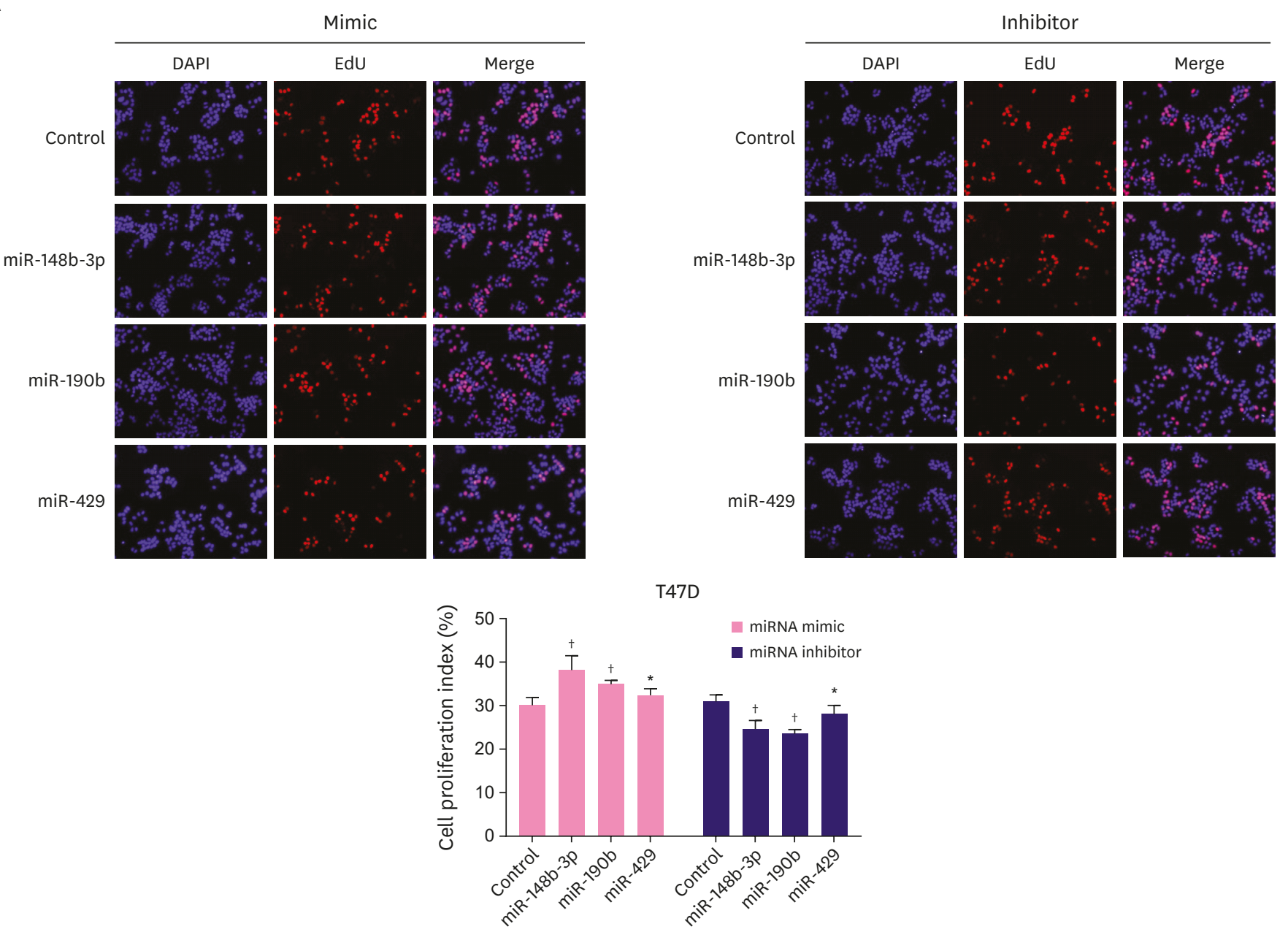

Figure 3. Effect of miR-148b-3p, miR-190b and miR-429 on breast cancer cell proliferation. The proliferative rate of T47D (A) and MDA-MB-468 (B) transfected with miRNA-control, miRNA mimics or miRNA inhibitors.

The data were representative of 3 technical repeats with the mean \pm standard deviation. $i R N A=$ microRNA; EdU $=5$-Ethynyl-2'-deoxyuridine.

${ }^{*} p<0.05,{ }^{\dagger} p<0.001$ vs. control using 1-way analysis of variance.

(continued to the next page) 
B

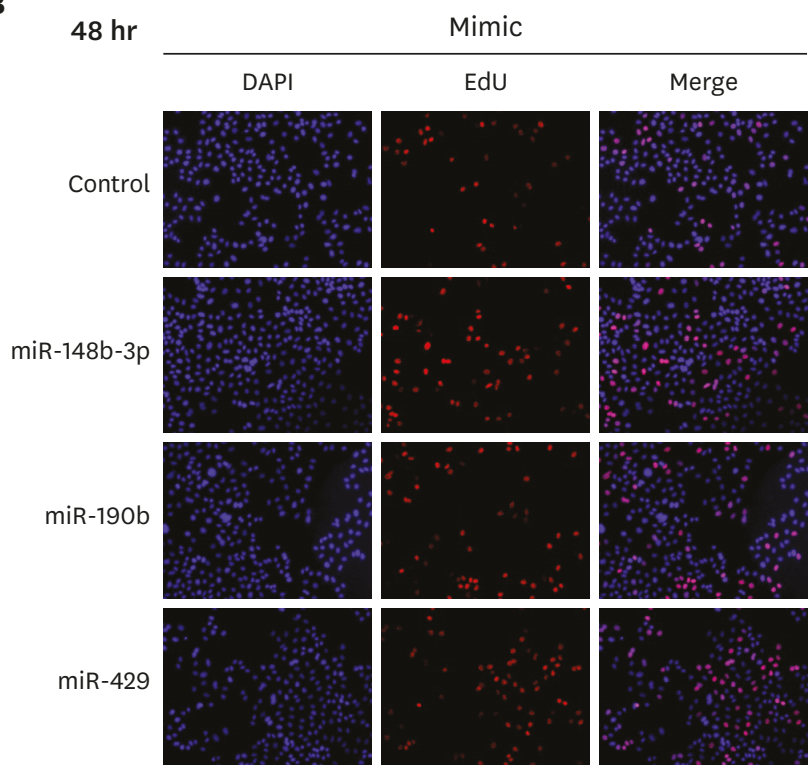

$48 \mathrm{hr}$
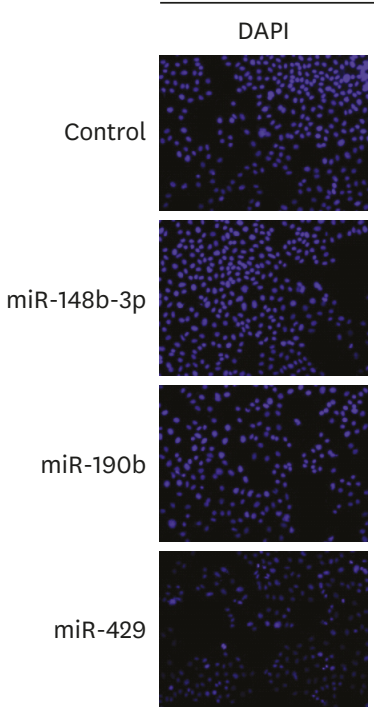

Inhibitor
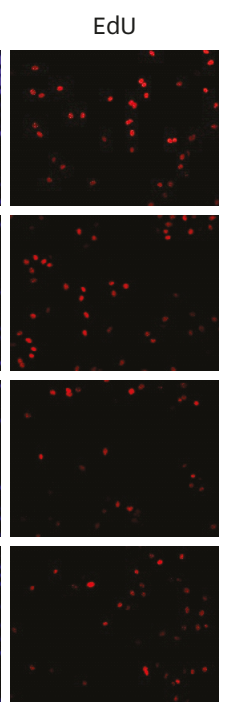
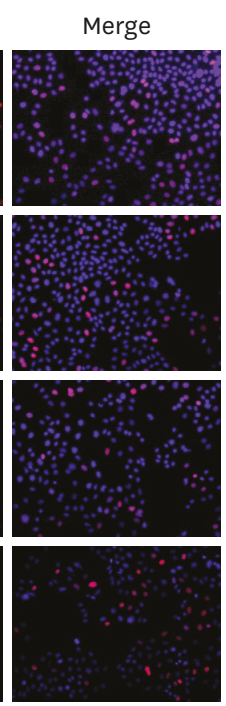

MDA-MB-468

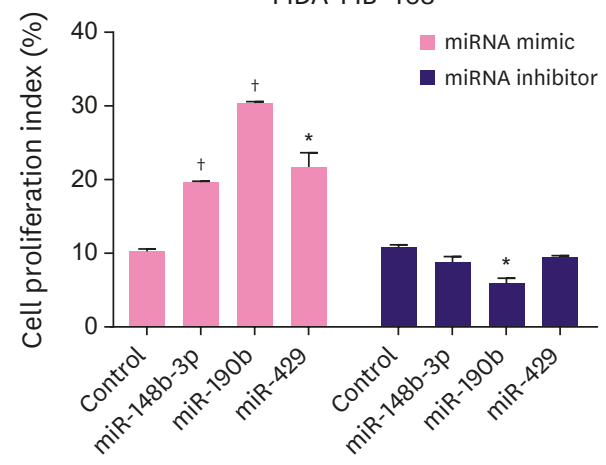

Figure 3. (Continued) Effect of miR-148b-3p, miR-190b and miR-429 on breast cancer cell proliferation. The proliferative rate of T47D (A) and MDA-MB-468 (B) transfected with miRNA-control, miRNA mimics or miRNA inhibitors.

The data were representative of 3 technical repeats with the mean \pm standard deviation. $\mathrm{R} R \mathrm{~A}=$ microRNA; EdU = 5-Ethynyl-2'-deoxyuridine.

${ }^{*} p<0.05,{ }^{\dagger} p<0.001$ vs. control using 1-way analysis of variance.

Effect of miR-148b-3p, miR-190b, and miR-429 on breast cancer cell apoptosis

To determine the effect of miR-148b-3p, miR-190b, and miR-429 on breast cancer cell apoptosis, we performed flow cytometric analysis. The results revealed the obvious decrease in the apoptosis of T47D cells transfected with miRNA mimics, while the apoptosis rate increased for the cells treated with miRNA inhibitors (Figure 4A). Western blotting results also confirmed that miR-148b-3p, miR-190b, and miR-429 inhibitors promoted PARP cleavage (Figure 4B).

Effect of miR-148b-3p, miR-190b, and miR-429 on breast cancer cell migration Wound healing assay was used to evaluate the effects of miR-148b-3p, miR-190b, and miR429 on the migratory behavior of MDA-MB-468 cells. As shown in Figure 5A and B, miRNA mimics significantly increased cell migration, while the miRNA inhibitors dramatically decreased the migration of treated cells. The analysis of the results of the above experiments showed that miR-190b exerted the most significant effects on cell proliferation, apoptosis, and migration. Therefore, we selected MDA-MB-231 cells with low expression of miR-190b for verification. We found that the overexpression of miR-190b dramatically decreased the migratory ability of these cells (Figure $\mathbf{5 C}$ ). To confirm the above observations, a transwell 
A

Mimic
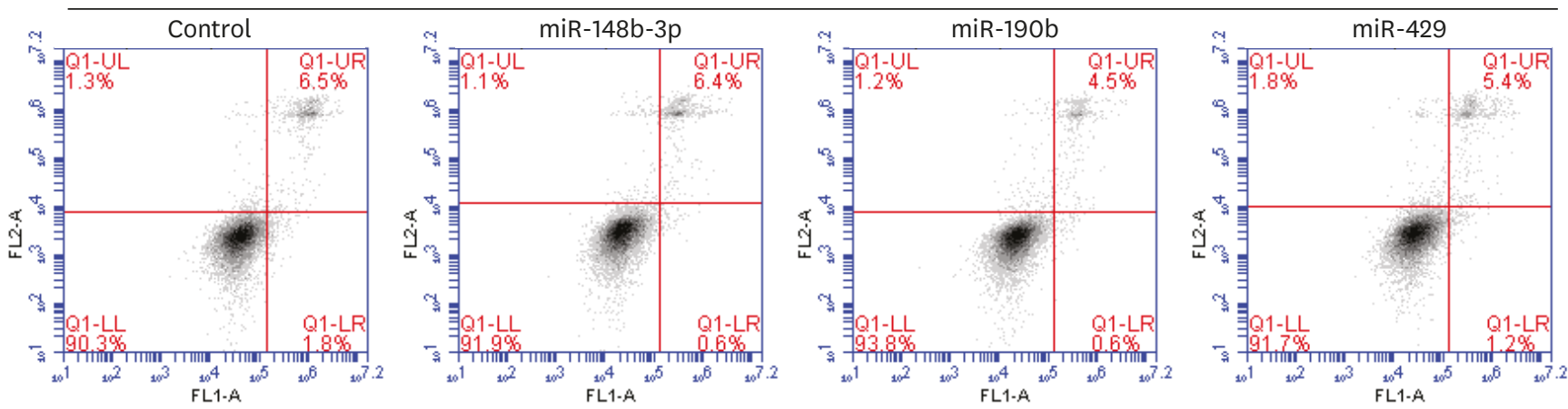

Inhibitor
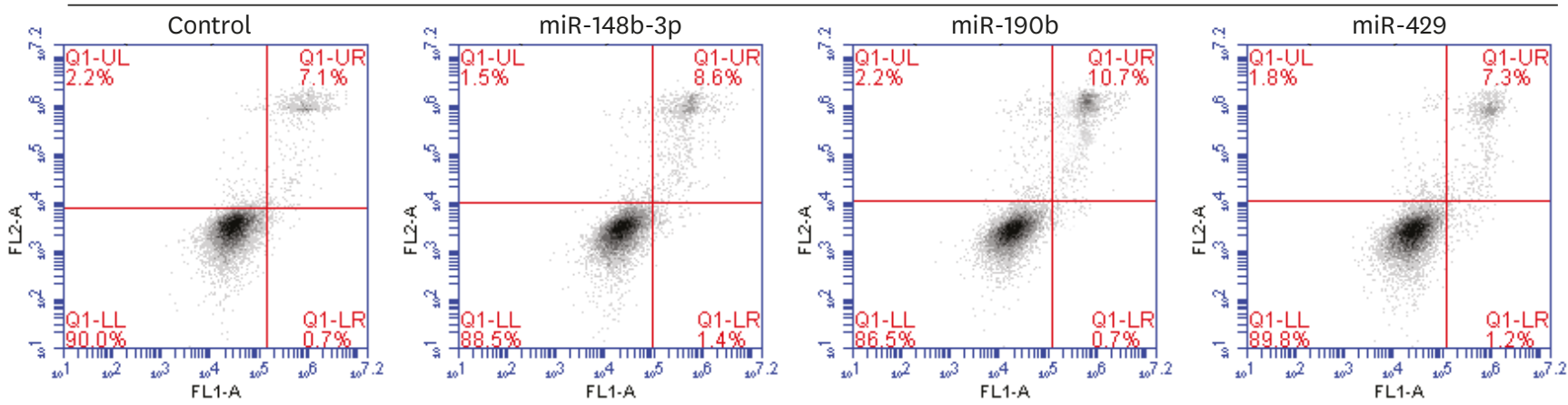

T47D

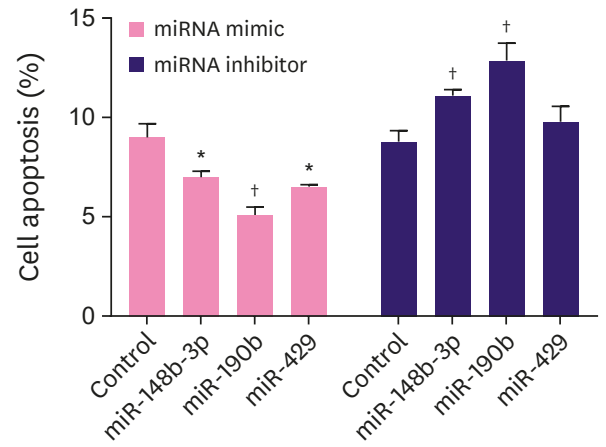

B

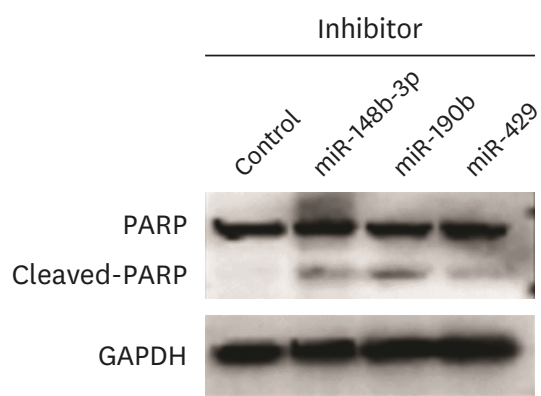

Figure 4. Effect of miR-148b-3p, miR-190b and miR-429 on breast cancer cell apoptosis. A. The influence of miRNAs on T47D cells apoptosis was evaluated by flow cytometry. B. The PARP and cleaved PARP protein levels were measured after transfection with miRNA inhibitors in T47D cells.

The data were representative of 3 technical repeats with the mean \pm standard deviation.

PARP = poly (ADP-ribose) polymerase; $\mathrm{miRNA}=$ microRNA; GAPDH = glyceraldehyde 3-phosphate dehydrogenase.

${ }^{*} p<0.05,{ }^{\dagger} p<0.01$ vs. control using 1-way analysis of variance. 
A

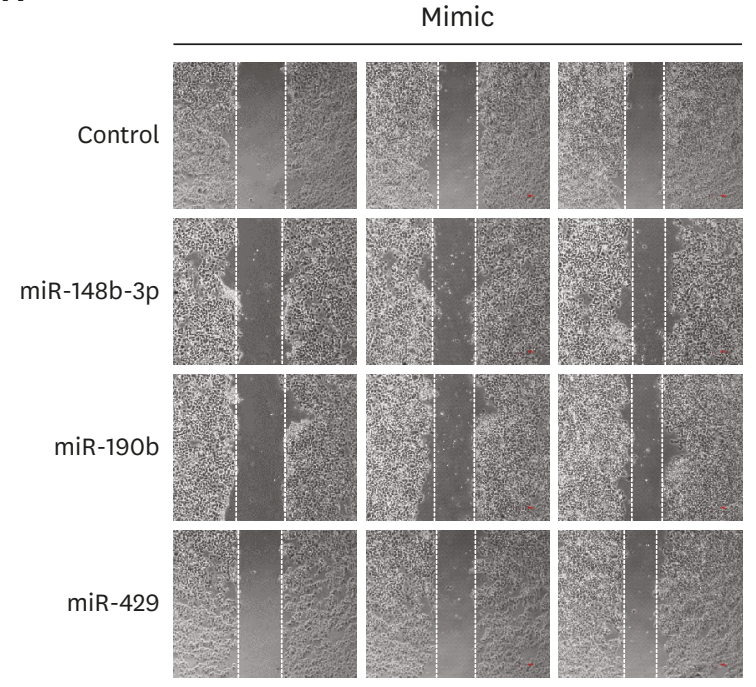

B

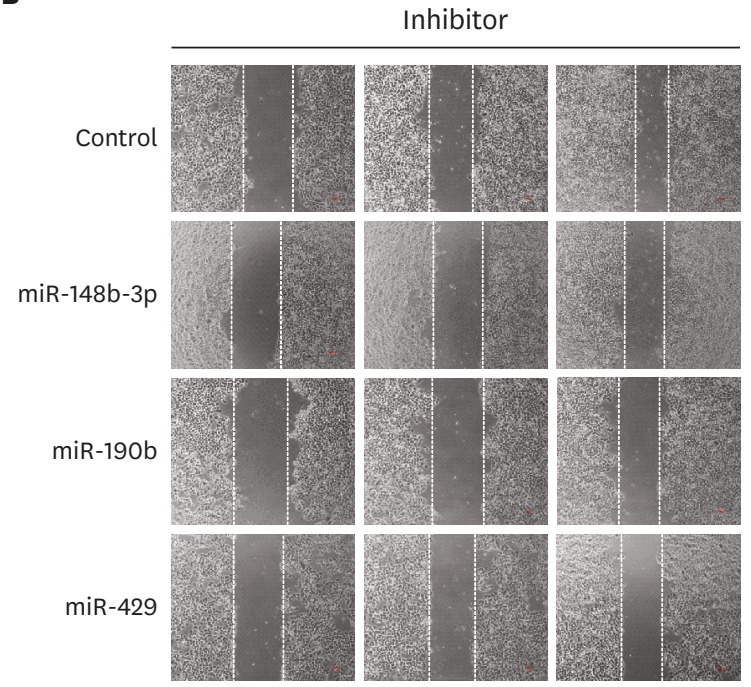

MDA-MB-468

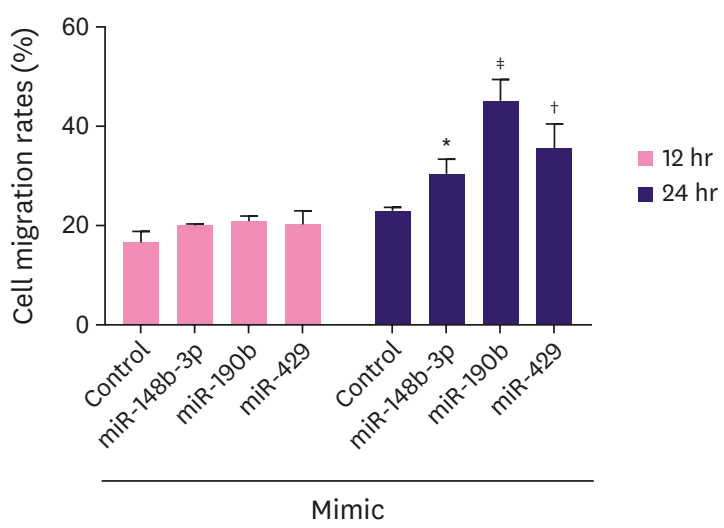

MDA-MB-468

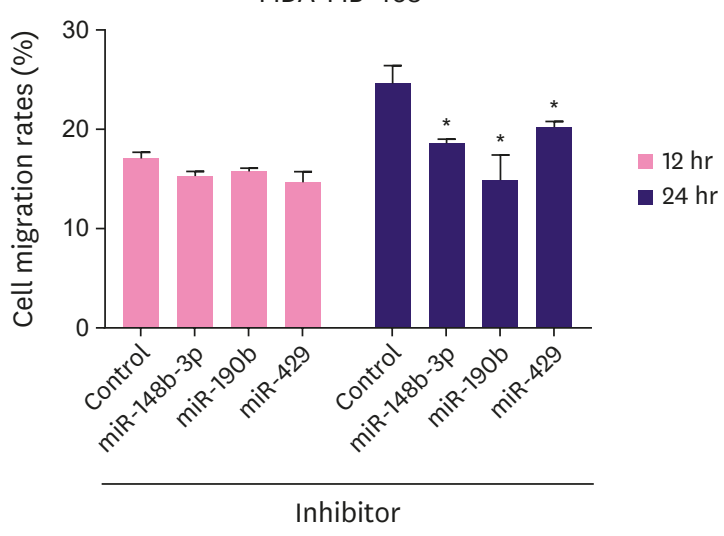

C

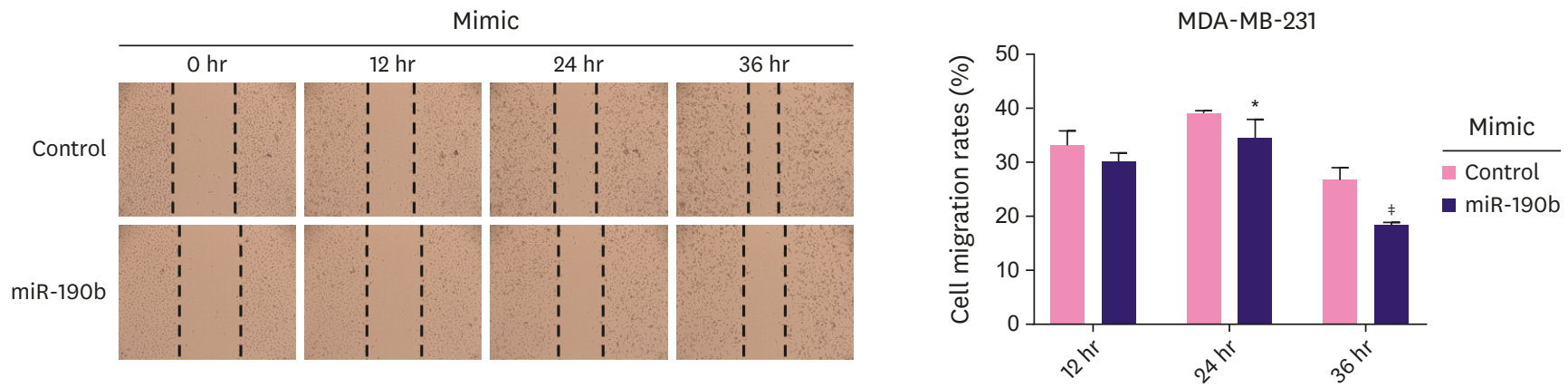

Figure 5. Effect of miR-148b-3p, miR-190b and miR-429 on breast cancer cell migration. The migration capacity of MDA-MB-468 cell transfected with miRNA mimics (A) or miRNA inhibitors (B) was detected by using wound-healing assay. (C) The capacity of MDA-MB-231 cell migration was decreased after treatment with miR-190b mimic based on the result of wound-healing assay. D. The ability of cell migration in MDA-MB-231 cells transfected with miR-190b mimic or inhibitor based on transwell. The data were representative of 3 technical repeats with the mean \pm standard deviation. miRNA $=$ microRNA.

${ }^{*} p<0.05,{ }^{\dagger} p<0.01$, and ${ }^{\ddagger} p<0.001$ vs. control using 1-way analysis of variance. 
D

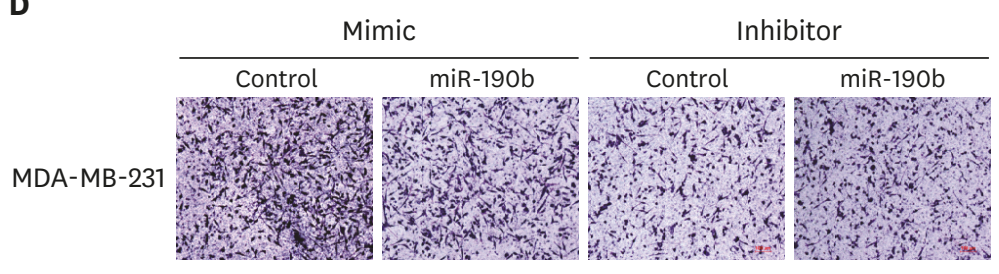

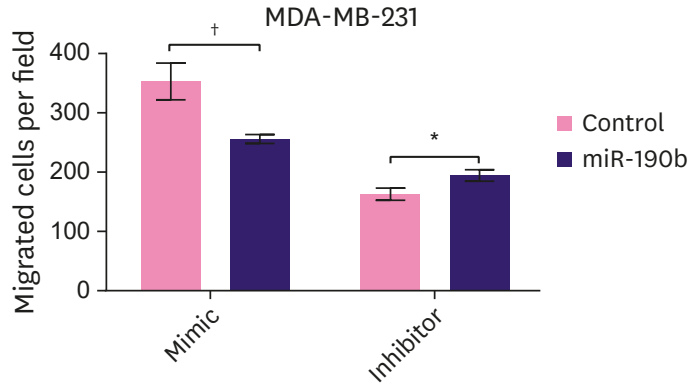

Figure 5. (Continued) Effect of miR-148b-3p, miR-190b and miR-429 on breast cancer cell migration. The migration capacity of MDA-MB-468 cell transfected with miRNA mimics (A) or miRNA inhibitors (B) was detected by using wound-healing assay. (C) The capacity of MDA-MB-231 cell migration was decreased after treatment with miR-190b mimic based on the result of wound-healing assay. D. The ability of cell migration in MDA-MB-231 cells transfected with miR-190b mimic or inhibitor based on transwell. The data were representative of 3 technical repeats with the mean \pm standard deviation. miRNA $=$ microRNA.

${ }^{*} p<0.05,{ }^{\dagger} p<0.01$, and ${ }^{\ddagger} p<0.001$ vs. control using 1-way analysis of variance.

assay was performed; the number of cells passing through the inserts was significantly lower in the miR-190b-overexpressing group than in the control group (Figure 5D).

\section{Target prediction and enrichment analysis}

To investigate the mechanisms underlying miR-148b-3p, miR-190b, and miR-429 functions, 9 online databases were scanned to predict target genes. We gathered target genes predicted by at least 4 algorithms to obtain 405 target genes for miR-148b-3p, 76 target genes for miR-190b, and 299 target genes for miR-429 (Supplementary Table 2). These genes were subjected to GO and pathway enrichment analyses (Supplementary Tables 3-5). The result revealed that the targets of candidate miRNAs were involved in several critical cancerrelated pathways, including transforming growth factor (TGF)-beta signaling pathway, Wnt signaling pathway, p53 pathway, p38 mitogen-activated protein kinase (MAPK) pathway and mammalian target of rapamycin (mTOR) signaling pathway (Figure 6).

\section{DISCUSSION}

At present, breast cancer is the most prevalent cancer among women and is the leading cause of cancer-related deaths among females [10]. Clinical data show that early diagnosis and treatment may be conducive for the cure and prognosis of patients and can reduce the suffering in breast cancer patients [3]. However, the lack of relevant clinical biomarkers with good sensitivity and specificity has limited the early diagnosis and prognosis of breast cancer. Previous studies have reported that miRNAs play vital roles in the initiation and progression of breast cancer and that certain miRNAs may act as potential diagnostic and prognostic biomarkers [11]. However, these miRNAs have not been widely exploited for clinical application, owing to the use of different profiling platforms. Some studies have employed microarray or RT-qPCR analyses, which generate signatures based on the available number of miRNAs $[12,13]$. Some other studies have included a limited number of patients and lacked effective clinical data [14].

TCGA is a large database of cancer and tumor genetic mapping programs sponsored by the U.S. government and includes data from more than 1,000 patients with breast cancer. In this study, we used the available data from TCGA and applied stringent filtering criteria to obtain highly sensitive and specific biomarkers. We first used the edgeR package of $R$ language ( $R$ 


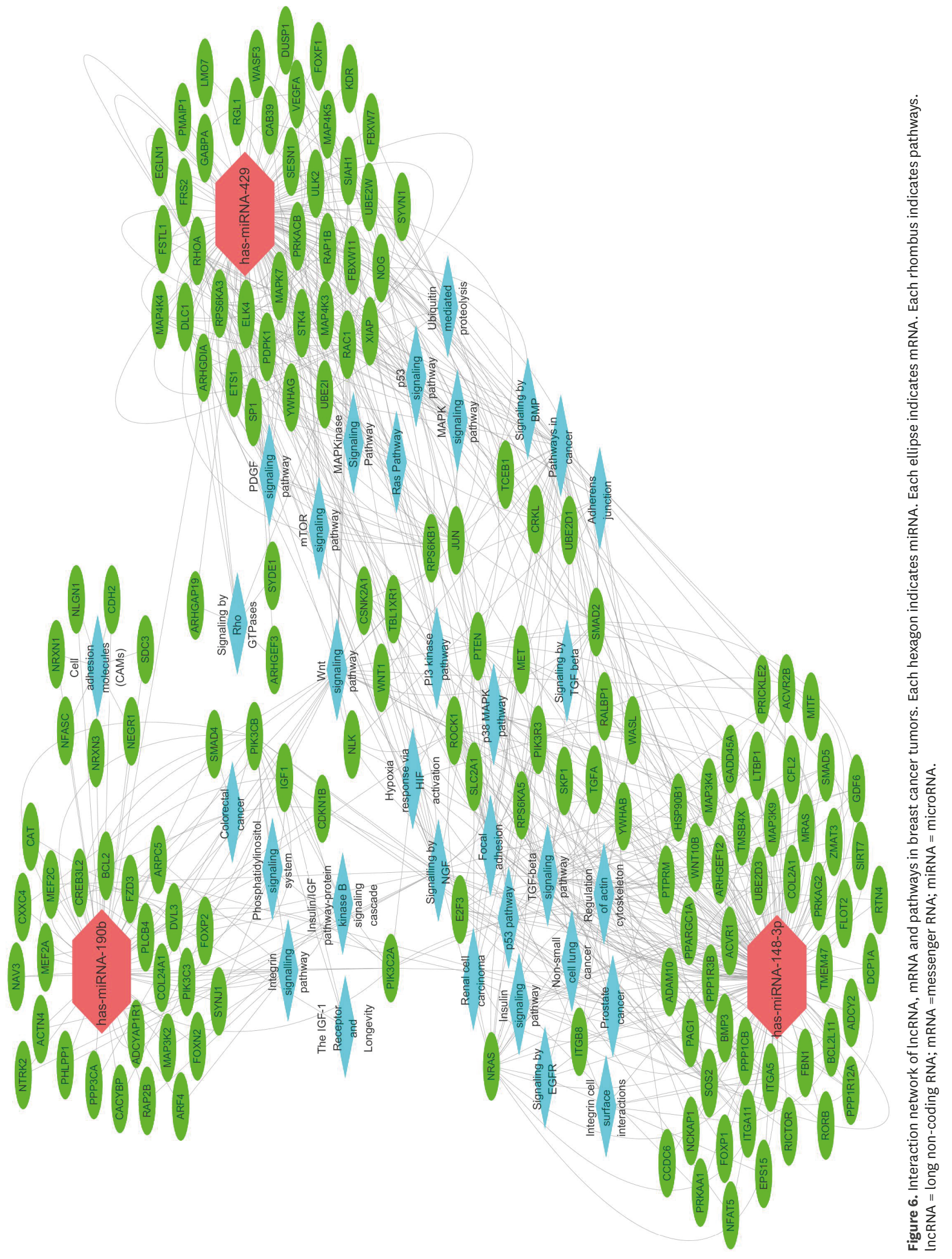


Foundation) to screen differentially expressed miRNAs, followed by the analysis of survival curves using the first 68 miRNAs according to the clinical information of patients with breast cancer. As a result, 13 miRNAs showed significant correlation with the survival time of patients. Furthermore, ROC curves were used to evaluate the accuracy of these 13 miRNAs as molecular markers for the diagnosis of breast cancer; 3 miRNAs with high specificity and sensitivity $(p<0.001$, AUC $>0.8$ ) were selected, including miR-148b-3p (AUC $=0.852 ; 95 \%$ CI, 0.819-0.885; $p<0.001$ ), miR-190b (AUC = 0.854; 95\% CI, 0.827-0.881; $p<0.001$ ), and miR-429 (AUC $=0.936 ; 95 \%$ CI, 0.915-0.957; $p<0.001$ ). The ROC curve of the combination of the 3 differentially expressed miRNAs showed an extremely high diagnostic accuracy with an AUC value of 0.950 ( $95 \% \mathrm{CI}, 0.930-0.971 ; p<0.001)$. Together, the data led us to conclude that the 3 miRNAs may serve as diagnostic and prognostic markers for breast cancer.

The miRNA screening process employed in the present study may be described as a layer-by-layer filtering method, which differs from the methods used in other studies that screened miRNAs with a certain index. For instance, Yin et al. [15] downloaded and collected miRNA and mRNA expression data from TCGA database, and identified the abnormally expressed miRNAs and mRNAs in breast cancer by comparing tumor samples with normal adjacent tissues, followed by the calculation of the Spearman's correlation coefficient to predict target genes and highlight the miRNAs associated with breast cancer. This study only involved differential expression, did not combine the survival time of patients, and had a large probability of false positives.

We studied the mechanism underlying the effects of the 3 selected miRNAs and found that the expression of miR-148b-3p, miR-190b, and miR-429 was high in TCGA database; however, no significant difference was observed in the expression of these 3 miRNAs between the cancer and normal groups in the validation set. The expression of miRNAs varied between different types of breast cancers, and as the number of samples we collected was insufficient, no significant difference was reported between groups; however, the changing trend in the expression patterns of these miRNAs was consistent with that observed in TCGA database. Similarly, some differences in expression patterns were also observed among different breast cancer cell lines. In comparison with the normal breast cell line MCF-10A, MCF-7, T47D, MDA-MB-468, and HCC1806 cells showed overexpression of miR-148b-3p, miR-190b, and miR-429, whereas the expression of these miRNAs was downregulated in MDA-MB-231 cells, consistent with the previously reported observations [16-18]. We carried out Kaplan-Meier analysis in different breast cancer subtypes based on TCGA database and found that the patients with high miR-190b expression had better OS than those with low miR-190b expression in luminal A, basal-like, and normal breast-like groups; however, the patients from luminal B and HER2 groups with high miR-190b expression had poor OS. These observations suggest that the biological and clinical implications of miRNAs may differ among breast cancer subtypes [16-18].

The results reported for the miRNA miR-148b-3p, encoded on chromosome 12q13.13, showed similarities as well as discrepancies with the previously reported data. Differences in experimental settings and materials may have contributed to different results. As observed herein, the expression of miR-148b-3p was shown to be upregulated in the blood plasma of patients with breast cancer [19] and was high in luminal samples [17]. However, another study showed that miR-148b expression was downregulated in the serum samples from patients with breast cancer [20]. miR-148b was identified as a relapse-associated miRNA and was shown to suppress breast cancer progression by targeting a series of cancer-related oncogenes [21]. These results suggest the complex role of miR-148b-3p that may be dependent on different tissue and cell types. Further functional studies are warranted to characterize the potential role of miR-148b-3p in breast cancer. 
Few studies have reported the implications of miR-190b expression in breast cancer. miR-190b was associated with estrogen-positive breast cancer [16] and hormone therapy resistance [22]. de Anda-Jáuregui et al. [23] recently identified miR-190b among 5 other miRNAs as a small set of miRNAs expressed in the breast cancer network; these authors proposed the important role of this mRNA set in the control of biological functions dysregulated in breast cancer. Naorem et al. [24] reported the dysregulated miRNAs, including miR-190b, in triple-negative breast cancer using a meta-analysis approach. However, none of these studies explored the role of miR-190b. In the present study, we reported that the overexpression of miR-190b may significantly promote the proliferation and migration of MDA-MB-468 and T47D cells and reduce the apoptosis of T47D cells. In contrast, Cizeron-Clairac et al. [16] showed that miR-190b may probably have no effect on cell proliferation. This discrepancy may be related to the differences in the transfection efficiency.

As a member of the miR-200 family, miR-429 plays several complex roles in the occurrence and development of cancer. miR-429 was overexpressed in MCF-7, T47D, MDA-MB-468, and HCC1806 cells but showed downregulated expression in MDA-MB-231 and SK-BR-3 cells, consistent with the results reported in previous studies $[18,25]$. We analyzed cell proliferation and migration and found that the overexpression of miR-429 could significantly promote the proliferation and migration of MDA-MB-468 and T47D cells. Some studies found that the re-expression of one or more miR-200 family members may reduce cell proliferation and migration [26,27]. However, miR-200 family members were also shown to enhance mammary tumor cell migration [28-30]. This discrepancy may be related to the differences in the cell lines and cancer subtypes analyzed. So, miRNAs were expressed in a cell type-specific and cancer subtype-specific manner.

To investigate the underlying mechanisms of action of miR-148b-3p, miR-190b, and miR-429, we used 9 online databases and predicted the target genes. Only the target genes predicted by at least 4 algorithms were chosen and subsequently used for $\mathrm{GO}$ and pathway enrichment analyses to explore the potential effects. The results revealed that the targets of these dysregulated miRNAs were involved in many critical cancer-related biological processes and pathways such as TGF-beta signaling pathway, Wnt signaling pathway, p53 pathway, p38 MAPK pathway, and mTOR signaling pathway. Thus, these miRNAs may not only act as biomarkers for breast cancer but may also be considered as targets for the development of new therapies.

To avoid the contingency caused by small sample size, this study included 1,083 breast cancer samples and 104 normal breast tissue samples from TCGA database. Therefore, the bias was reduced, and the screened miRNAs were more representative candidates. The present study, however, has several limitations. First, although the basic cellular function of the 3 selected miRNAs was validated herein, the exact target genes and pathways and the mechanism underlying their effects on the survival time of patients were incompletely understood. Second, the expression profiling of these 3 miRNAs was based on clinical tissues. Circulating miRNAs have several advantages as biomarkers; hence, it is necessary to detect the expression of these 3 miRNAs in the peripheral blood of patients. At the same time, it is important to expand clinical samples, improve clinical information, and analyze their correlation with pathological index of patients.

In summary, we identified that miR-148b-3p, miR-190b, and miR-429 may serve as potential breast cancer biomarkers using high-throughput data analysis from TCGA dataset. We also discovered that these 3 miRNAs affect breast cancer cell proliferation, apoptosis, and 
migration. However, multicenter studies with large sample sizes are warranted to validate our findings and further investigation of the roles of these 3 miRNAs is necessary.

\section{SUPPLEMENTARY MATERIALS}

\section{Supplementary Table 1}

Differentially expressed miRNAs

Click here to view

\section{Supplementary Table 2}

Target genes for 3 miRNAs

Click here to view

\section{Supplementary Table 3}

GO functional annotation and pathway analyses of has-miR-148b-3p

Click here to view

\section{Supplementary Table 4}

GO functional annotation and pathway analyses of has-miR-190b

Click here to view

\section{Supplementary Table 5}

GO functional annotation and pathway analyses of has-miR-429

Click here to view

\section{Supplementary Figure 1}

Kaplan-Meier survival curves showing different overall survival in groups of patients with low and high miRNAs expression.

Click here to view

\section{Supplementary Figure 2}

Expression level of has-miR-148b-3p (A), has-miR-190b (B), has-miR-429 (C) in different breast cancer subtypes.

click here to view

\section{Supplementary Figure 3}

Prognostic value of has-miR-148b-3p (A), has-miR-190b (B), and has-miR-429 (C) in breast cancer subtypes varies among different subtypes.

Click here to view 


\section{Supplementary Figure 4}

In MDA-MB-468 (A) and T47D (B) cell lines, expression of miRNAs was significantly increased by miRNAs mimics but was decreased by miRNAs inhibitors compared with the corresponding control.

Click here to view

\section{REFERENCES}

1. Schneider AP 2nd, Zainer CM, Kubat CK, Mullen NK, Windisch AK. The breast cancer epidemic: 10 facts. Linacre Q 2014;81:244-77. PUBMED | CROSSREF

2. Fan L, Strasser-Weippl K, Li JJ, St Louis J, Finkelstein DM, Yu KD, et al. Breast cancer in China. Lancet Oncol 2014;15:e279-89.

PUBMED | CROSSREF

3. Liu YR, Jiang YZ, Xu XE, Hu X, Yu KD, Shao ZM. Comprehensive transcriptome profiling reveals multigene signatures in triple-negative breast cancer. Clin Cancer Res 2016;22:1653-62. PUBMED | CROSSREF

4. Carthew RW, Sontheimer EJ. Origins and mechanisms of miRNAs and siRNAs. Cell 2009;136:642-55. PUBMED | CROSSREF

5. Tutar Y. miRNA and cancer; computational and experimental approaches. Curr Pharm Biotechnol 2014;15:429. PUBMED | CROSSREF

6. Yang Y, Qu A, Zhao R, Hua M, Zhang X, Dong Z, et al. Genome-wide identification of a novel miRNAbased signature to predict recurrence in patients with gastric cancer. Mol Oncol 2018;12:2072-84. PUBMED | CROSSREF

7. Harquail J, Benzina S, Robichaud GA. MicroRNAs and breast cancer malignancy: an overview of miRNAregulated cancer processes leading to metastasis. Cancer Biomark 2012;11:269-80. PUBMED | CROSSREF

8. Iorio MV, Casalini P, Piovan C, Braccioli L, Tagliabue E. Breast cancer and microRNAs: therapeutic impact. Breast 2011;20 Suppl 3:S63-70. PUBMED | CROSSREF

9. Li HY, Liang JL, Kuo YL, Lee HH, Calkins MJ, Chang HT, et al. miR-105/93-3p promotes chemoresistance and circulating miR-105/93-3p acts as a diagnostic biomarker for triple negative breast cancer. Breast Cancer Res 2017;19:133. PUBMED | CROSSREF

10. Siegel RL, Miller KD, Jemal A. Cancer statistics, 2016. CA Cancer J Clin 2016;66:7-30. PUBMED | CROSSREF

11. Gao Y, Cai Q, Huang Y, Li S, Yang H, Sun L, et al. MicroRNA-21 as a potential diagnostic biomarker for breast cancer patients: a pooled analysis of individual studies. Oncotarget 2016;7:34498-506. PUBMED

12. Chen X, Wang YW, Zhu WJ, Li Y, Liu L, Yin G, et al. A 4-microRNA signature predicts lymph node metastasis and prognosis in breast cancer. Hum Pathol 2018;76:122-32. PUBMED | CROSSREF

13. Yao L, Liu Y, Cao Z, Li J, Huang Y, Hu X, et al. MicroRNA-493 is a prognostic factor in triple-negative breast cancer. Cancer Sci 2018;109:2294-301. PUBMED | CROSSREF

14. Xiong DD, Lv J, Wei KL, Feng ZB, Chen JT, Liu KC, et al. A nine-miRNA signature as a potential diagnostic marker for breast carcinoma: an integrated study of 1,110 cases. Oncol Rep 2017;37:3297-304. PUBMED | CROSSREF

15. Yin Y, Shen C, Xie P, Cheng Z, Zhu Q. Construction of an initial microRNA regulation network in breast invasive carcinoma by bioinformatics analysis. Breast 2016;26:1-10. PUBMED | CROSSREF

16. Cizeron-Clairac G, Lallemand F, Vacher S, Lidereau R, Bieche I, Callens C. MiR-190b, the highest upregulated miRNA in ER $\alpha$-positive compared to ER $\alpha$-negative breast tumors, a new biomarker in breast cancers? BMC Cancer 2015;15:499. PUBMED | CROSSREF 
17. Aure MR, Leivonen SK, Fleischer T, Zhu Q, Overgaard J, Alsner J, et al. Individual and combined effects of DNA methylation and copy number alterations on miRNA expression in breast tumors. Genome Biol 2013;14:R126. PUBMED | CROSSREF

18. Li D, Wang H, Song H, Xu H, Zhao B, Wu C, et al. The microRNAs miR-200b-3p and miR-429-5p target the LIMK1/CFL1 pathway to inhibit growth and motility of breast cancer cells. Oncotarget 2017;8:85276-89. PUBMED

19. Cuk K, Zucknick M, Heil J, Madhavan D, Schott S, Turchinovich A, et al. Circulating microRNAs in plasma as early detection markers for breast cancer. Int J Cancer 2013;132:1602-12. PUBMED | CROSSREF

20. Mangolini A, Ferracin M, Zanzi MV, Saccenti E, Ebnaof SO, Poma VV, et al. Diagnostic and prognostic microRNAs in the serum of breast cancer patients measured by droplet digital PCR. Biomark Res 2015;3:12. PUBMED | CROSSREF

21. Cimino D, De Pittà C, Orso F, Zampini M, Casara S, Penna E, et al. miR148b is a major coordinator of breast cancer progression in a relapse-associated microRNA signature by targeting ITGA5, ROCK1, PIK3CA, NRAS, and CSF1. FASEB J 2013;27:1223-35. PUBMED | CROSSREF

22. Joshi T, Elias D, Stenvang J, Alves CL, Teng F, Lyng MB, et al. Integrative analysis of miRNA and gene expression reveals regulatory networks in tamoxifen-resistant breast cancer. Oncotarget 2016;7:57239-53. PUBMED | CROSSREF

23. de Anda-Jáuregui G, Espinal-Enríquez J, Drago-García D, Hernández-Lemus E. Nonredundant, highly connected microRNAs control functionality in breast cancer networks. Int J Genomics 2018;2018:9585383. PUBMED | CROSSREF

24. Naorem LD, Muthaiyan M, Venkatesan A. Identification of dysregulated miRNAs in triple negative breast cancer: a meta-analysis approach. J Cell Physiol. Epub 2018 Nov 29. https://doi.org/10.1002/jcp.27839. PUBMED | CROSSREF

25. Patel N, Garikapati KR, Makani VK, Nair AD, Vangara N, Bhadra U, et al. Regulating BMI1 expression via miRNAs promote mesenchymal to epithelial transition (MET) and sensitizes breast cancer cell to chemotherapeutic drug. PLoS One 2018;13:e0190245. PUBMED | CROSSREF

26. Tryndyak VP, Beland FA, Pogribny IP. E-cadherin transcriptional down-regulation by epigenetic and microRNA-200 family alterations is related to mesenchymal and drug-resistant phenotypes in human breast cancer cells. Int J Cancer 2010;126:2575-83. PUBMED

27. Burk U, Schubert J, Wellner U, Schmalhofer O, Vincan E, Spaderna S, et al. A reciprocal repression between ZEB1 and members of the miR-200 family promotes EMT and invasion in cancer cells. EMBO Rep 2008;9:582-9. PUBMED | CROSSREF

28. Choi SK, Kim HS, Jin T, Hwang EH, Jung M, Moon WK. Overexpression of the miR-141/200c cluster promotes the migratory and invasive ability of triple-negative breast cancer cells through the activation of the FAK and PI3K/AKT signaling pathways by secreting VEGF-A. BMC Cancer 2016;16:570. PUBMED | CROSSREF

29. Yu SJ, Hu JY, Kuang XY, Luo JM, Hou YF, Di GH, et al. MicroRNA-200a promotes anoikis resistance and metastasis by targeting YAP1 in human breast cancer. Clin Cancer Res 2013;19:1389-99. PUBMED | CROSSREF

30. Jin T, Suk Kim H, Ki Choi S, Hye Hwang E, Woo J, Suk Ryu H, et al. microRNA-200c/141 upregulates SerpinB2 to promote breast cancer cell metastasis and reduce patient survival. Oncotarget 2017;8:32769-82. PUBMED 